\title{
Full counting statistics of transport through two-channel Coulomb blockade systems
}

\author{
Shi-Kuan Wang, Hujun Jiao, Feng Li, and Xin-Qi Li* \\ State Key Laboratory for Superlattices and Microstructures, Institute of Semiconduct., Chinese Academy of Sciences, \\ P.O. Box 912, Beijing 100083, China
}

YiJing Yan

Department of Chemistry, Hong Kong University of Science and Technology, Kowloon, Hong Kong

(Received 28 March 2007; revised manuscript received 19 June 2007; published 14 September 2007)

\begin{abstract}
A mesoscopic Coulomb blockade system with two transport channels is studied in terms of full counting statistics. It is found that the shot noise and skewness are crucially affected by the quantum mechanical interference. In particular, the super-Poisson behavior can be induced as a consequence of constructive interference, and can be understood by the formation of effective fast-and-slow transport channels. Dephasing and finite temperature effects are carried out together with physical interpretations.
\end{abstract}

DOI: 10.1103/PhysRevB.76.125416

PACS number(s): 73.23.-b, 73.50.Td

\section{INTRODUCTION}

Rather than average current, the current fluctuations in mesoscopic transport can sometimes provide deep insight into the nature of transport mechanisms. ${ }^{1}$ A fascinating theoretical approach, known as full counting statistics (FCS) theory, 2,3 can simultaneously yield all the statistical cumulants of the number of transferred charges (i.e., all zerofrequency current-correlation functions). Experimentally, the real-time counting statistics has been carried out in transport through quantum dots, ${ }^{4}$ representing a crucial achievement of being able to count individual electron tunnel events.

For charge transport at very low transmission, the uncorrelated transmission events are Poisson processes. However, the Fermi-Dirac statistics together with the possible manybody Coulomb interaction usually enhances correlation among the transport electrons, and thereby results in subPoisson noise. ${ }^{5}$ It is thus of interest to examine mechanisms that can lead to super-Poisson-noise behavior, since the current fluctuations can be used in reverse to gain insight into the nature of unusual transport mechanisms. The mechanisms proposed so far for the super-Poisson noise include double electron charge transfer by Andreev reflection in normal-superconductor junction, ${ }^{6,7}$ multiple electron charge transfer by multiple Andreev reflections in superconductornormal-superconductor system, ${ }^{8-11}$ dynamical channel blockade, ${ }^{12-14}$ dynamical spin blockade, ${ }^{15}$ bistability, ${ }^{16}$ cotunneling, ${ }^{17,18}$ electron-phonon interaction in shuttle system, ${ }^{19}$ and decoherence in mesoscopic coherent population trapping system. ${ }^{20}$

In this work we consider a relatively simple system, say, electronic transport through a Coulomb blockade system with two identical transport channels, which can be realized experimentally by transport through two adjacent levels in a single quantum dot (QD), ${ }^{21}$ or through two QDs in parallel. $^{22,23}$ This type of setup itself is of particular interest, since it is an analog of the optical double-slit interferometer. In this context the underlying quantum interference and phase accumulations through QDs have been the subjects of intensive studies. ${ }^{24-26}$ Our present study will be placed at the level of FCS, from which a number of interesting effects of quantum interference on current fluctuations will be revealed.
The paper is organized as follows. In Sec. II we present the model description together with the underlying physical considerations and assumptions. In Sec. III we outline the main idea and procedures to obtain the particle-number resolved master equation and to relate it with the FCS formulation. Since the separate details have been published elsewhere, ${ }^{27-29}$ for the sake of saving space we would like to omit the detailed derivation in this work and suggest to refer to the previous publications. In Secs. IV and V we carry out the main results of this work, including the average current, the shot noise and the skewness, as well as the dephasing effects on them. Interference effects, in particular, the interference induced super-Poisson behavior, will be highlighted and explained. To optimally manifest the effects of interference and dephasing on FCS, in these two sections we restrict our study to zero temperature. In Sec. VI we extend the study to finite temperatures, where the interplay between the (quantum) fast-and-slow channel modulated stochastic process and the thermal fluctuations is found to play an important role in affecting the current noise and skewness. Finally, in Sec. VII we summarize the work.

\section{MODEL DESCRIPTION}

The transport through a two-channel Coulomb blockade system is governed by the Hamiltonian

$$
\begin{gathered}
H=H_{D}+H_{\text {leads }}+H_{T}, \\
H_{D}=E_{1} d_{1}^{\dagger} d_{1}+E_{2} d_{2}^{\dagger} d_{2}+U n_{1} n_{2}, \\
H_{\text {leads }}=\sum_{k}\left(\varepsilon_{L k} c_{L k}^{\dagger} c_{L k}+\varepsilon_{R k} c_{R k}^{\dagger} c_{R k}\right), \\
H_{T}=\sum_{j k}\left[\Omega_{j L} d_{j}^{\dagger} c_{L k}+\Omega_{j R} d_{j}^{\dagger} c_{R k}+\text { H.c. }\right] .
\end{gathered}
$$

Here $c_{L k, R k}^{\dagger}\left(c_{L k, R k}\right)$ and $d_{j}^{\dagger}\left(d_{j}\right)$ are the electron creation (annihilation) operators, for the electrode reservoirs and central dot states, respectively. The two channels are characterized by states with energy levels $E_{1}$ and $E_{2}$. Couplings of these 
two dot states to the electrodes are described by $\Omega_{j L(R)}$, or $\Gamma_{L, R}^{j}=2 \pi g_{L, R}\left|\Omega_{j L, j R}\right|^{2}$, for latter use. Here $g_{L, R}$ are the density of states (DOS) of the electron reservoirs. To manifest maximally the quantum interference effect, we shall focus on two identical transmission paths. This can be accomplished by assuming equal and energy independent coupling strengths of the two dot states with the left and right electrodes, i.e., $\left|\Omega_{1 L(R)}\right|=\left|\Omega_{2 L(R)}\right|=\Omega_{L(R)}$, and $\Gamma_{L(R)}^{1}=\Gamma_{L(R)}^{2}=\Gamma_{L(R)}$. To address the quantum interference between transmissions through the two channels, the relative phase difference is significant. Physically, the phase difference contains the phase accumulation of spatial motion from the electrode to dot, particularly in the presence of magnetic vector potential (i.e., the Aharanov-Bohm effect), as well as the phase changes associated with transmission through quantum dots. ${ }^{25,26}$ Nevertheless, in this work we would like to adopt a phenomenological way to account for all these phase accumulations, by choosing $\Omega_{1 L}=\Omega_{2 L}$, and $\eta=\Omega_{1 R} / \Omega_{2 R}$. Here $\eta$ can be regarded as a relative phase parameter. Note that the alternative gauge, say, $\Omega_{1 R}=\Omega_{2 R}$ and $\eta=\Omega_{1 L} / \Omega_{2 L}$, does not affect the final results. ${ }^{21}$ In this paper, we also assumed $\eta= \pm 1$, i.e., only complete constructive and destructive interferences are considered.

In the above Hamiltonian we omitted the spin indices, thus did not explicitly write out the on-site Coulomb interaction terms, and only left $U n_{1} n_{2}$ to describe Coulomb interaction between electrons in the different dot states. In this work, unless explicit specification, our study will be restricted to the strong Coulomb blockade regime, which only allows for three available occupation states, i.e., $|0\rangle,|1\rangle$, and $|2\rangle$, corresponding to, respectively, empty dot state, and states with one electron on either $E_{1}$ or $E_{2}$.

\section{FULL COUNTING STATISTICS FORMULATION}

As pointed out in Ref. 29, the pioneering work $^{2,3}$ and a few other approaches developed later ${ }^{10,31,32}$ are largely restricted to addressing the FCS of noninteracting electrons. While Ref. 29 developed an elegant theory of FCS for mesoscopic systems in strong Coulomb blockade limit, however, the system's internal quantum coherence did not enter it since the theory was constructed on the basis of classical stochastic processes. It is thus advantageous to formulate an approach of being able to account for both the internal quantum coherence and the many-body Coulomb interaction on equal footing. Although such type of approach has been described and applied to coherent and interacting systems, ${ }^{33,34}$ we would like to reformulate it for the sake of completeness, based on the particle-number-resolved master equation at finite temperatures. ${ }^{27,28}$

To relate with our earlier work, ${ }^{27,28}$ we rename $H_{S} \equiv H_{D}$, $H_{B} \equiv H_{\text {leads }}$, and reexpress $H^{\prime} \equiv H_{T}$ as

$$
\begin{gathered}
H^{\prime}=H_{T}=\sum_{j=1}^{2}\left[d_{j}^{\dagger} F_{j}+\text { H.c. }\right], \\
F_{j}=\sum_{k} \Omega_{j L} c_{L k}+\sum_{k} \Omega_{j R} c_{R k}=f_{L j}+f_{R j} .
\end{gathered}
$$

Regarding $H^{\prime}$ as perturbation, the second-order cumulant expansion leads to a formal master equation for the system's reduced density matrix as follows: $:^{27,28}$

$$
\dot{\rho}(t)=-i \mathcal{L} \rho(t)-\int_{0}^{t} d \tau\left\langle\mathcal{L}^{\prime}(t) \mathcal{G}(t, \tau) \mathcal{L}^{\prime}(\tau) \mathcal{G}^{\dagger}(t, \tau)\right\rangle \rho(t)
$$

Here the Liouvillian superoperators are defined as $\mathcal{L} \rho$ $=\left[H_{S}, \rho\right], \mathcal{L}^{\prime} \rho=\left[H^{\prime}, \rho\right]$, and $\mathcal{G}(t, \tau)(\cdots)=G(t, \tau)(\cdots) G^{\dagger}(t, \tau)$, with $G(t, \tau)$ the usual propagator associated with system Hamiltonian $H_{S}$. The reduced density matrix $\rho(t)$ $=\operatorname{Tr}_{B}\left[\rho_{T}(t)\right]$, and $\langle\cdots\rangle=\operatorname{Tr}_{B}\left[\cdots \rho_{B}\right]$ with $\rho_{B}$ the density matrix of the electron reservoirs.

The trace in Eq. (3) is over all the electrode degrees of freedom. To achieve the FCS of current fluctuations, we would like to keep track of the records of electron numbers emitted from the source lead $\left(n_{1}\right)$ and arrived at the drain lead $\left(n_{2}\right)$. We therefore classify the Hilbert space of the reservoirs as follows: $B^{\left(n_{1}, n_{2}\right)}=B_{L}^{\left(n_{1}\right)} \otimes B_{R}^{\left(n_{2}\right)}$. The entire Hilbert space of electron reservoirs is thus decomposed as $B=$ $\oplus_{n_{1}, n_{2}} B^{\left(n_{1}, n_{2}\right)}$.

With this classification the average over states in the entire bath Hilbert space in Eq. (3) is replaced with the average over states in the subspace $B^{\left(n_{1}, n_{2}\right)}$, leading to a conditional master equation

$$
\begin{aligned}
\dot{\rho}^{\left(n_{1}, n_{2}\right)}(t)= & -i \mathcal{L} \rho^{\left(n_{1}, n_{2}\right)}(t)-\int_{0}^{t} d \tau \operatorname{Tr}_{B}\left(n_{1}, n_{2}\right) \\
& \times\left[\mathcal{L}^{\prime}(t) \mathcal{G}(t, \tau) \mathcal{L}^{\prime}(\tau) \mathcal{G}^{\dagger}(t, \tau) \rho_{T}(t)\right] .
\end{aligned}
$$

Here, $\rho^{\left(n_{1}, n_{2}\right)}(t)=\operatorname{Tr}_{B^{\left(n_{1}, n_{2}\right)}}\left[\rho_{T}(t)\right]$ is the reduced density matrix of the central system conditioned by the electron numbers emitted from the source lead $\left(n_{1}\right)$ and arrived at the drain lead $\left(n_{2}\right)$ until time $t$.

To proceed, following Ref. 28, two physical considerations are further implemented: (i) Instead of the conventional Born approximation for the entire density matrix $\rho_{T}(t) \simeq \rho(t) \otimes \rho_{B}$, we propose the ansatz $\rho_{T}(t) \simeq \sum_{n_{1}, n_{2}} \rho^{\left(n_{1}, n_{2}\right)}$ $\times(t) \otimes \rho_{B}^{\left(n_{1}, n_{2}\right)}$, where $\rho_{B}^{\left(n_{1}, n_{2}\right)}(t)$ is the density operator of the electron reservoirs associated with $n_{1}$ electrons emitted from the source and $n_{2}$ electrons entered the drain. The orthogonality between reservoirs states in different subspaces leads to the term selection from the entire density operator $\rho_{T}$. (ii) Due to the closed nature of the transport circuit, the extra electrons that entered the drain will flow back into the source via the external circuit. Also the rapid relaxation processes in the reservoirs will quickly bring the reservoirs to local thermal equilibrium characterized by the chemical potentials. As a consequence, after the state selection procedure, the electron reservoir density matrices $\rho^{\left(n_{1}, n_{2}\right)}$ should be replaced by $\rho_{B}^{(0)}$.

Further use of the Markov-Redfield approximation leads Eq. (4) to an explicit form

$$
\dot{\rho}^{\left(n_{1}, n_{2}\right)}=-i \mathcal{L} \rho^{\left(n_{1}, n_{2}\right)}-\frac{1}{2} \mathcal{R}_{1} \rho^{\left(n_{1}, n_{2}\right)},
$$




$$
\begin{aligned}
\mathcal{R}_{1} \rho^{\left(n_{1}, n_{2}\right)}= & \sum_{j}\left[d_{j}^{\dagger} A_{j}^{(-)} \rho^{\left(n_{1}, n_{2}\right)}+\rho^{\left(n_{1}, n_{2}\right)} A_{j}^{(+)} d_{j}^{\dagger}-A_{L j}^{(-)} \rho^{\left(n_{1}-1, n_{2}\right)} d_{j}^{\dagger}\right. \\
& -d_{j}^{\dagger} \rho^{\left(n_{1}+1, n_{2}\right)} A_{L j}^{(+)}-A_{R j}^{(-)} \rho^{\left(n_{1}, n_{2}-1\right)} d_{j}^{\dagger} \\
& \left.-d_{j}^{\dagger} \rho^{\left(n_{1}, n_{2}+1\right)} A_{R j}^{(+)}\right]+ \text {H.c. }
\end{aligned}
$$

Here $A_{\alpha j}^{(+)}=\Sigma_{i} \widetilde{C}_{\alpha i j}^{(+)}(+\mathcal{L}) d_{i}, \quad A_{\alpha j}^{(-)}=\Sigma_{i} \widetilde{C}_{\alpha j i}^{(-)}(-\mathcal{L}) d_{i}, \quad$ and $\quad A_{j}^{( \pm)}$ $=\sum_{\alpha=L, R} A_{\alpha j}^{( \pm)}$. The spectral functions are defined in terms of the Fourier transform of the reservoir correlation functions, i.e., $\widetilde{C}_{\alpha i j}^{( \pm)}( \pm \mathcal{L})=\int_{-\infty}^{\infty} d t C_{\alpha i j}^{( \pm)}(t) e^{ \pm i \mathcal{L} t}$. The reservoir correlators $\operatorname{read}\left\langle f_{\alpha i}^{\dagger}(t) f_{\alpha j}(\tau)\right\rangle=C_{\alpha i j}^{(+)}(t-\tau)$, and $\left\langle f_{\alpha i}(t) f_{\alpha j}^{\dagger}(\tau)\right\rangle=C_{\alpha i j}^{(-)}(t-\tau)$. Here $\langle\cdots\rangle$ stands for $\operatorname{Tr}_{B}\left[\cdots \rho_{B}^{(0)}\right]$, with the usual meaning of thermal average. Obviously, $\left\langle F_{i}^{\dagger}(t) F_{j}(\tau)\right\rangle=C_{i j}^{(+)}(t-\tau)$ $=\Sigma_{\alpha=L, R} C_{\alpha i j}^{(+)}(t-\tau)$, and $\left\langle F_{i}(t) F_{j}^{\dagger}(\tau)\right\rangle=C_{i j}^{(-)}(t-\tau)=\Sigma_{\alpha=L, R} C_{\alpha i j}^{(-)}(t$ $-\tau)$. For the sake of brevity, the explicit expressions of the reservoir correlation functions, the corresponding spectral functions, and $A_{\alpha j}^{( \pm)}$are ignored here, and are presented alternatively in Appendix A.

At this stage, we would like to make a few remarks as follows: (i) The above particle-number-resolved master equation is applicable to finite temperatures, which is an extension of Gurvitz and Prager's wave function approach. ${ }^{30} \mathrm{At}$ finite temperature and small bias voltage, the electrons can jump back from the right reservoir to the central dots, and from the central dots to the left reservoir. These processes are described, respectively, by the $\rho^{\left(n_{1}+1\right)}$ and $\rho^{\left(n_{2}+1\right)}$ terms in the above equations, whereas these terms are absent in Gurvitz and Prager's equations. ${ }^{30}$ (ii) The second-order cumulant expansion of $H^{\prime}$ restricts the applicability to the regime of sequential tunneling. However, generalization to higher order expansion of $H^{\prime}$ (Refs. 35 and 36) and self-consistent corrections $^{37}$ are possible. The corresponding FCS version is an interesting subject for future work. (iii) The above $\left(n_{1}, n_{2}\right)$-resolved master equation generalizes the result in Ref. 28, from counting at one junction to counting at two junctions. Further generalization to multiterminal setup is straightforward, following precisely the same treatment. (iv) The connection of the particle-number-resolved density matrix with the distribution function of FCS is obvious, i.e., $P\left(n_{1}, n_{2}, t\right)=\operatorname{Tr}\left[\rho(t)^{\left(n_{1}, n_{2}\right)}\right]$, where the trace is over the central system states. From this distribution function, all orders of cumulants of transmission electrons can be calculated.

In practice, instead of obtaining the distribution function from the solution of the particle-number-resolved master equation, a more efficient method is the cumulant generating function (CGF) technique. In the following study, we only consider single counting statistics. That is, we only keep $n_{2}$, after making summation over $n_{1}$. Multiple counting statistics in multiterminal setup follows the same technique.

Mathematically, the CGF is introduced as ${ }^{29}$

$$
e^{-F(\chi)}=\sum_{n} P(n, t) e^{i n \chi}
$$

Here $\chi$ corresponds to the so-called counting field. Based on the CGF, the $k$ th cumulant reads $C_{k}=-\left.\left(-i \partial_{\chi}\right)^{k} F(\chi)\right|_{\chi=0}$. For instance, the first two cumulants give rise to the mean value of the transmitted electron numbers $C_{1}=\bar{n}$, and the variance
$C_{2}=\overline{n^{2}}-\bar{n}^{2}$; the third cumulant (skewness), $C_{3}=\overline{(n-\bar{n})^{3}}$, characterizes the asymmetry of the distribution. Here, $\overline{(\cdots)}$ $=\Sigma_{n}(\cdots) P(n, t)$. Moreover, the cumulants are straightforwardly related to the transport characteristics, e.g., the average current by $I=e C_{1} / t$, and the zero-frequency shot noise by $S=2 e^{2} C_{2} / t$. The Fano factor is defined as $F=C_{2} / C_{1}$, which represents the amplitude of current fluctuations, with $F>1$ indicating a super-Poisson fluctuation, and $F<1$ a subPoisson process.

The generating function can be calculated as follows: Define $S(\chi, t)=\Sigma_{n} \rho^{(n)}(t) e^{i n \chi}$. Obviously, $e^{-F(\chi)}=\operatorname{Tr}[S(\chi, t)]$. Let us reexpress the particle-number-resolved master equation formally as

$$
\dot{\rho}^{(n)}=A \rho^{(n)}+C \rho^{(n+1)}+D \rho^{(n-1)},
$$

then $S(\chi, t)$ satisfies

$$
\dot{S}=A S+e^{-i \chi} C S+e^{i \chi} D S \equiv \mathcal{L}_{\chi} S .
$$

The formal solution reads $S(\chi, t)=e^{\mathcal{L} \chi^{t}} S(\chi, 0)$. In the lowfrequency limit, the counting time is much longer than the time of tunneling through the system. One can prove, ${ }^{20,29,33,34}$ that $F(\chi)=-\lambda_{1}(\chi) t$, where $\lambda_{1}(\chi)$ is the eigenvalue of $\mathcal{L}_{\chi}$, and satisfies the condition $\left.\lambda_{1}(\chi)\right|_{\chi \rightarrow 0} \rightarrow 0$.

The above FCS formalism based on Eq. (5) is an extension of Gurvitz and Prager's approach ${ }^{34}$ from zero temperature to finite temperatures, and an extension of Ref. 29 to include internal quantum coherence. In the following, we apply this approach to the system described in Sec. II, first restricting to zero temperature in order to more clearly reveal the pure quantum-interference induced phenomena and dephasing effect, then carrying out the results at finite temperatures.

\section{FULL COUNTING STATISTICS ANALYSIS}

For the specific model described in Sec. II, in the strong Coulomb blockade regime and at zero temperature, the matrix element form of Eq. (5) reads

$$
\begin{gathered}
\dot{\rho}_{00}^{\left(n_{2}\right)}=-2 \Gamma_{L} \rho_{00}^{\left(n_{2}\right)}+\Gamma_{R}\left(\rho_{11}^{\left(n_{2}-1\right)}+\rho_{22}^{\left(n_{2}-1\right)}\right) \\
+\eta \Gamma_{R}\left(\rho_{12}^{\left(n_{2}-1\right)}+\rho_{21}^{\left(n_{2}-1\right)}\right), \\
\dot{\rho}_{11}^{\left(n_{2}\right)}=\Gamma_{L} \rho_{00}^{\left(n_{2}\right)}-\Gamma_{R} \rho_{11}^{\left(n_{2}\right)}-\eta \frac{\Gamma_{R}}{2}\left(\rho_{12}^{\left(n_{2}\right)}+\rho_{21}^{\left(n_{2}\right)}\right), \\
\dot{\rho}_{22}^{\left(n_{2}\right)}=\Gamma_{L} \rho_{00}^{\left(n_{2}\right)}-\Gamma_{R} \rho_{22}^{\left(n_{2}\right)}-\eta \frac{\Gamma_{R}}{2}\left(\rho_{12}^{\left(n_{2}\right)}+\rho_{21}^{\left(n_{2}\right)}\right), \\
\dot{\rho}_{12}^{\left(n_{2}\right)}=i \delta \epsilon \rho_{12}^{\left(n_{2}\right)}+\Gamma_{L} \rho_{00}^{\left(n_{2}\right)}-\Gamma_{R} \rho_{12}^{\left(n_{2}\right)}-\eta \frac{\Gamma_{R}}{2}\left(\rho_{11}^{\left(n_{2}\right)}+\rho_{22}^{\left(n_{2}\right)}\right) .
\end{gathered}
$$

Here we have summed $n_{1}$ and remained only $n_{2}$, indicating the mere study of FCS of the electrons that entered the drain reservoir. For clarity, we have denoted the level spacing by $\delta \epsilon=E 2-E 1$, and choose the reference of zero energy such 

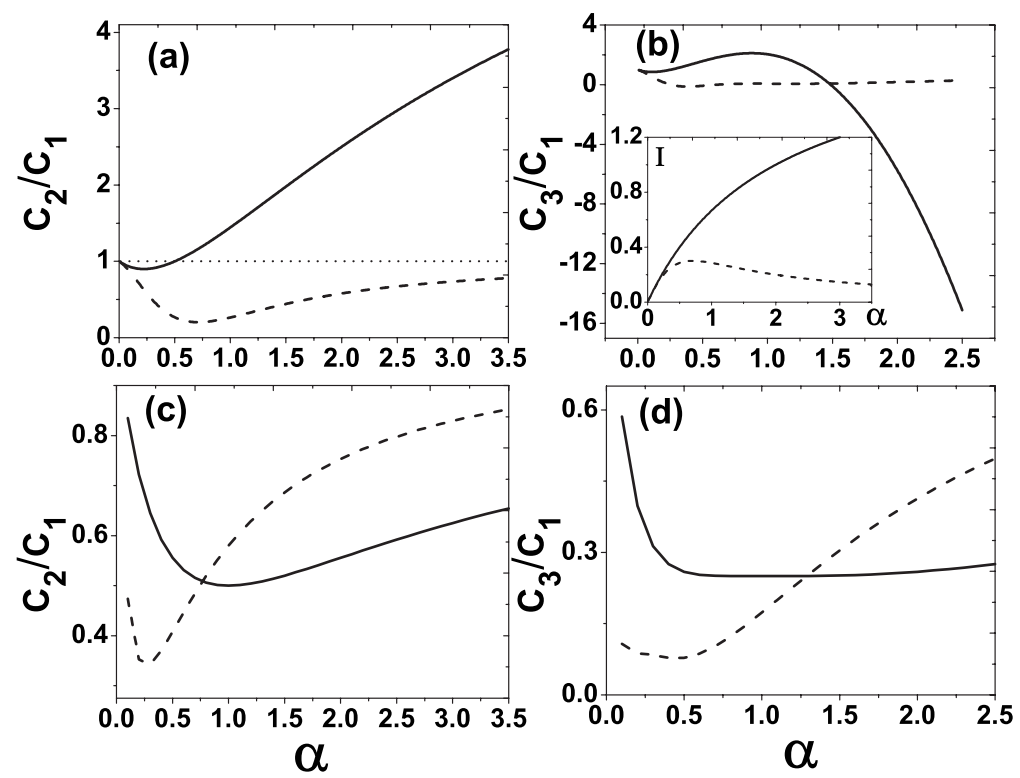

FIG. 1. First three cumulants of the zero-frequency current fluctuations. The solid and dashed curves display the results for constructive $(\eta=1)$ and destructive $(\eta=-1)$ interferences, respectively. The variable $\alpha=\Gamma_{R} / \Gamma_{L}$ characterizes the asymmetry of dot-state couplings to the left and right electrodes. (a) and (b) show the Fano factor and skewness for strong Coulomb blockade system, while (c) and (d) for system allowing double occupancy, i.e., $U=0.0$, as a comparison. The inset of (b) plots the average current $I=e C_{1} / t$, with the convention $e=1$. In the calculation $\Gamma_{L}=1.0 \delta \epsilon$ was assumed.

that $E 2=\delta \epsilon / 2$, and $E 1=-\delta \epsilon / 2$. In the derivation of Eqs. (9), we assumed that $E_{1,2}$ are inside the window of bias voltage, i.e., $\mu_{L}>E_{1,2}>\mu_{R}$, and $U$ is infinite. In Ref. 21 the same Eqs. (9) were derived by using a wave function approach, where the interference effects on current and frequencydependent noise spectrum were studied. In the following we present a FCS study for this same system.

Performing a discrete Fourier transformation $\Sigma_{n_{2}} e^{i n_{2} \chi}$ to Eqs. (9), we obtain

$$
\mathcal{L}_{\chi}=\left(\begin{array}{ccccc}
-2 \Gamma_{L} & \Gamma_{R} e^{i \chi} & \Gamma_{R} e^{i \chi} & \eta \Gamma_{R} e^{i \chi} & \eta \Gamma_{R} e^{i \chi} \\
\Gamma_{L} & -\Gamma_{R} & 0 & -\eta \frac{\Gamma_{R}}{2} & -\eta \frac{\Gamma_{R}}{2} \\
\Gamma_{L} & 0 & -\Gamma_{R} & -\eta \frac{\Gamma_{R}}{2} & -\eta \frac{\Gamma_{R}}{2} \\
\Gamma_{L} & -\eta \frac{\Gamma_{R}}{2} & -\eta \frac{\Gamma_{R}}{2} & i \delta \epsilon-\Gamma_{R} & 0 \\
\Gamma_{L} & -\eta \frac{\Gamma_{R}}{2} & -\eta \frac{\Gamma_{R}}{2} & 0 & -i \delta \epsilon-\Gamma_{R}
\end{array}\right) .
$$

According to the definition of the cumulants we can express $\lambda_{1}(\chi)$ as

$$
\lambda_{1}(\chi)=\frac{1}{t} \sum_{k=1}^{\infty} C_{k} \frac{(i \chi)^{k}}{k !} .
$$

Then insert the above expansion into $\left|\lambda_{1}(\chi) I-\mathcal{L}_{\chi}\right|=0$, and expand this determinant in series of $(i \chi)^{k}$. Since the value of $i \chi$ is arbitrary, we can obtain $C_{k}$ by setting the coefficients of $(i \chi)^{k}$ equal to zero and solving them sequentially. ${ }^{20}$ Analytic expressions of the first two cumulants are accordingly obtained as

$$
C_{1}=\frac{2 \Gamma_{L} \Gamma_{R} \delta \epsilon^{2}}{2 \Gamma_{L}\left(\delta \epsilon^{2}-(\eta-1) \Gamma_{R}^{2}\right)+\Gamma_{R} \delta \epsilon^{2}}
$$

$$
C_{2}=\frac{\delta \epsilon^{4} \Gamma_{R}^{2}+4 \Gamma_{L}^{2}\left[\delta \epsilon^{4}+2 \delta \epsilon^{2} \eta \Gamma_{R}^{2}+(\eta-1)^{2} \Gamma_{R}^{4}\right]}{\left[2 \Gamma_{L}\left(\delta \epsilon^{2}-(\eta-1) \Gamma_{R}^{2}\right)+\Gamma_{R} \delta \epsilon^{2}\right]^{3}} 2 \Gamma_{L} \Gamma_{R} \delta \epsilon^{2},
$$

while the higher order cumulants can be instead carried out numerically, to avoid their lengthy expressions.

In Fig. 1 the first three cumulants of transport current are displayed. It is of interest to note that in the Coulomb blockade regime a super-Poisson noise is developed by the constructive interference between the two paths (i.e., $\eta=+1)$. This is clearly shown by the solid curve in Fig. 1(a). With the increase of the coupling asymmetry (i.e., $\alpha=\Gamma_{R} / \Gamma_{L}$ ), the super-Poisson feature will be more evident. In contrast, for destructive interference $(\eta=-1)$, the current fluctuation is sub-Poissonian, as plotted by the dashed curve in Fig. 1(a).

Another intriguing finding is that the super- and subPoisson characteristics are associated with different behaviors of the skewness $C_{3} / C_{1}$, as shown in Fig. 1(b). For destructive interference, the skewness is approximately zero, meanwhile for constructive interference, transition of the skewness from (small) positive to (large) negative values takes place, by increasing the coupling asymmetry $(\alpha)$. As is well known for photon counting statistics in quantum optics, the skewness (both its magnitude and sign) provides further information for the counting statistics, beyond the secondorder cumulant. As a comparison, the results of noninteracting system are plotted in Figs. 1(c) and 1(d), where neither the super-Poisson noise nor the negative skewness is found.

To understand better the above super-Poisson behavior, below we present an analysis in terms of fast-and-slow transport channels. Let us introduce an alternative representation for the double-dot states, with the corresponding electronic operators $f_{1}=\left(\Omega_{L} d_{1}+\Omega_{R} d_{2}\right) / \sqrt{\Omega_{L}^{2}+\Omega_{R}^{2}}$, and $f_{2}=\left(\Omega_{L} d_{2}\right.$ $\left.-\Omega_{R} d_{1}\right) / \sqrt{\Omega_{L}^{2}+\Omega_{R}^{2}}$, as well as the state energies $E_{1 / 2}$ $=\mp \delta \epsilon / 2$. In such representation the entire Hamiltonian is reexpressed as 

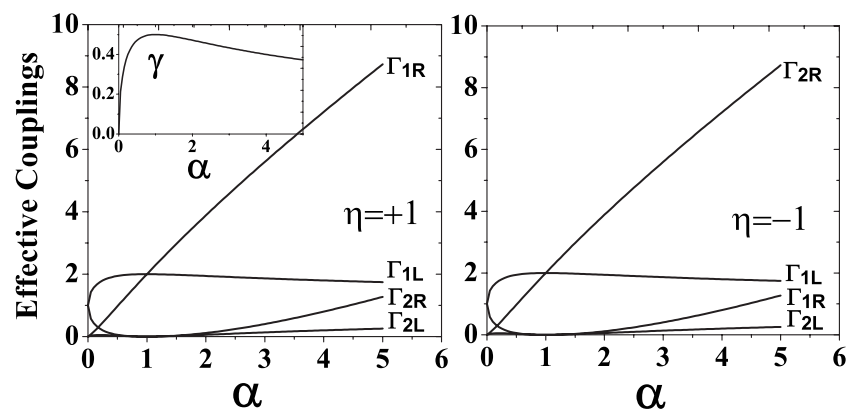

FIG. 2. Effective couplings of the dot states to the electrodes in the transformed state representation. It is found that effective fastand-slow transport channels are evaluated for the constructive interference $(\eta=1)$, which is contrasted remarkably with the destructive interference $(\eta=-1)$. Inset: effective coupling between the transformed dot states. Parameters $\alpha=\Gamma_{R} / \Gamma_{L}$ and $\Gamma_{L}=\delta \epsilon=1.0$.

$$
\begin{aligned}
H= & \widetilde{E}_{1} f_{1}^{\dagger} f_{1}+\widetilde{E}_{2} f_{2}^{\dagger} f_{2}+\gamma\left(f_{1}^{\dagger} f_{2}+f_{2}^{\dagger} f_{1}\right)+\sum_{k}\left[\widetilde{\Omega}_{1 L} f_{1}^{\dagger} a_{L k}\right. \\
& \left.+\widetilde{\Omega}_{1 R} f_{1}^{\dagger} a_{R k}+\text { H.c. }\right]+\sum_{k}\left[\widetilde{\Omega}_{2 L} f_{2}^{\dagger} a_{L k}+\widetilde{\Omega}_{2 R} f_{2}^{\dagger} a_{R k}+\text { H.c. }\right] .
\end{aligned}
$$

Note that the strong Coulomb blockade, rather than being explicitly described in this Hamiltonian, is reflected alternatively by the single occupation of the two dot states. For the sake of brevity, explicit expressions for the coupling between the new dot states, and their couplings to the electrodes are presented in Appendix B.

In the new state representation, the formation of the fastand-slow transport channels is demonstrated in Fig. 2: (i) for constructive interference $(\eta=+1)$, increasing $\alpha=\Gamma_{R} / \Gamma_{L}$ can lead to $\Gamma_{1 L}>\Gamma_{2 L}$, and $\Gamma_{1 R} \gg \Gamma_{2 R}$; (ii) for destructive interference $(\eta=-1)$, however, increasing $\alpha=\Gamma_{R} / \Gamma_{L}$ leads to $\Gamma_{1 L}$ $>\Gamma_{2 L}$, but $\Gamma_{2 R} \gg \Gamma_{1 R}$. Obviously, in the former case, effective fast-and-slow transport channels are evaluated, but cannot in the second case. Such fast-and-slow transport channels will lead to bunching behavior, and result in the super-Poisson noise. $^{12-15}$

\section{DEPHASING EFFECT}

Dephasing between the two interfering paths originated from the random fluctuations of $E_{1}$ and $E_{2}$. This may be caused in general by uncontrollable complex environments. Alternatively, for the setup of transport through two quantum dots in parallel, a controllable dephasing mechanism can be implemented experimentally by performing the "which-way" detection using a nearby quantum point contact (QPC). ${ }^{24} \mathrm{As}$ a model description, the effect of which-way detection is described by the following Lindblad-type terms ${ }^{34,38,39}$

$$
L_{\phi} \rho^{\left(n_{2}\right)} L_{\phi}^{\dagger}-\frac{1}{2} L_{\phi}^{\dagger} L_{\phi} \rho^{\left(n_{2}\right)}-\frac{1}{2} \rho^{\left(n_{2}\right)} L_{\phi}^{\dagger} L_{\phi},
$$

where the jump operator $L_{\phi}=\sqrt{\Gamma_{d}}|1\rangle\langle 1|$. Explicitly, the which-way detection induced dephasing rate can be carried

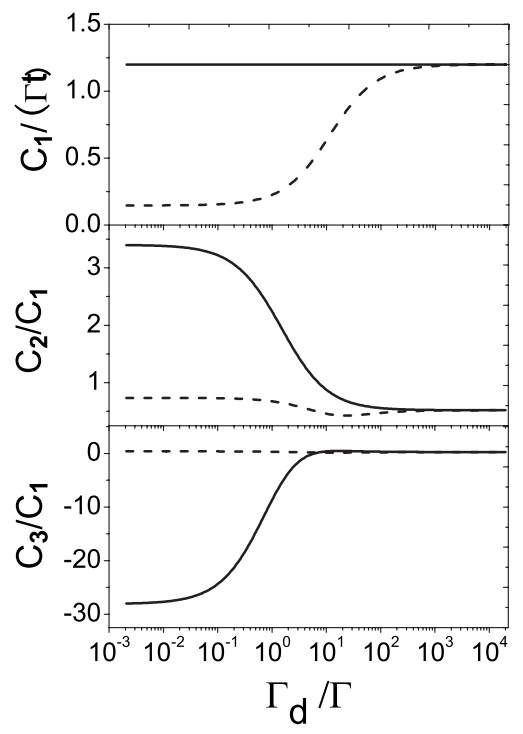

FIG. 3. Dephasing effects on the first three cumulants, which show a continuous transition from quantum interfering to classical noninterfering transports. The solid and dashed curves display results for the constructive $(\eta=1)$ and destructive $(\eta=-1)$ interferences, respectively. Parameters $\alpha=\Gamma_{R} / \Gamma_{L}=3.0$ and $\Gamma_{L}=\delta \epsilon=\Gamma$.

out as follows: ${ }^{38} \Gamma_{d}=\left(\sqrt{T}-\sqrt{T^{\prime}}\right)^{2} V_{d} / 2 \pi$, where $V_{d}$ is the bias voltage across the QPC, and $T$ and $T^{\prime}$ are the QPC-electron transmission probabilities, depending on which dot the electron passes through in the double-dot interferometer. We carry out the matrix elements of Eq. (14) in the state basis $\{|0\rangle,|1\rangle,|2\rangle\}$, and insert them into the right hand side of Eq. (9). The dephasing effects on the FCS can then be calculated straightforwardly.

Figure 3 shows the dephasing effects on the first three cumulants, where the solid and dashed curves correspond to the constructive $(\eta=1)$ and destructive $(\eta=-1)$ interferences, respectively. Interestingly, for the constructively interfering transport $(\eta=1)$, dephasing does not influence the transport current, see the solid line in Fig. 3(a). This is in remarkable contrast with the result of double-slit optical interference, where the constructively interfering intensity is four times of the intensity of the individual path (slit), while the noninterfering intensity is simply two times of the singleslit intensity. This essential difference originated from the multiple forward-and-backward scattering between the dot states and the electrodes in the case of electron transport. However, also for $\eta=1$, the second and third cumulants (i.e., $C_{2}$ and $C_{3}$ ) sensitively depend on the dephasing strength. In particular, dephasing would cause a transition from superPoisson to sub-Poisson processes, meanwhile the skewness $\left(C_{3}\right)$ changes from negative value to zero. The present analysis clearly shows that the super-Poisson current fluctuation is a consequence of the constructive interference. For destructively interfering transport $(\eta=-1)$, the almost vanished transport current will be restored by dephasing, whereas the shot noise and skewness approximately do not change with dephasing. 


\section{FINITE TEMPERATURE EFFECT}

At zero temperature, the Eqs. (9) we obtained on the basis of quantum statistical mechanics coincides with the one derived by Gurvitz and Prager based on a novel wave function approach. However, our approach is rather naturally capable of dealing with finite temperature effect. At finite temperature, calculating Eq. (5) in the state basis $\{|0\rangle,|1\rangle,|2\rangle\}$ results in

$$
\begin{aligned}
\dot{\rho}_{00}^{\left(n_{2}\right)}= & -\left[\Gamma_{L} n_{L}^{(+)}(E 1)+\Gamma_{R} n_{R}^{(+)}(E 1)+\Gamma_{L} n_{L}^{(+)}(E 2)+\Gamma_{R} n_{R}^{(+)}\right. \\
& \times(E 2)] \rho_{00}^{\left(n_{2}\right)}+\Gamma_{L} n_{L}^{(-)}(E 1) \rho_{11}^{\left(n_{2}\right)}+\Gamma_{R} n_{R}^{(-)}(E 1) \rho_{11}^{\left(n_{2}-1\right)} \\
+ & \Gamma_{L} n_{L}^{(-)}(E 2) \rho_{22}^{\left(n_{2}\right)}+\Gamma_{R} n_{R}^{(-)}(E 2) \rho_{22}^{\left(n_{2}-1\right)}+\frac{1}{2} \Gamma_{L}\left[n_{L}^{(-)}(E 2)\right. \\
+ & \left.n_{L}^{(-)}(E 1)\right]\left(\rho_{12}^{\left(n_{2}\right)}+\rho_{21}^{\left(n_{2}\right)}\right)+\frac{\eta}{2} \Gamma_{R}\left[n_{R}^{(-)}(E 2)+n_{R}^{(-)}(E 1)\right] \\
& \times\left(\rho_{12}^{\left(n_{2}-1\right)}+\rho_{21}^{\left(n_{2}-1\right)}\right), \\
\dot{\rho}_{11}^{\left(n_{2}\right)}= & \Gamma_{L} n_{L}^{(+)}(E 1) \rho_{00}^{\left(n_{2}\right)}+\Gamma_{R} n_{R}^{(+)}(E 1) \rho_{00}^{\left(n_{2}+1\right)}-\left[\Gamma_{L} n_{L}^{(-)}(E 1)\right. \\
& \left.+\Gamma_{R} n_{R}^{(-)}(E 1)\right] \rho_{11}^{\left(n_{2}\right)}-\frac{\Gamma_{L}}{2} n_{L}^{(-)}(E 2)\left(\rho_{12}^{\left(n_{2}\right)}+\rho_{21}^{\left(n_{2}\right)}\right) \\
& -\eta \frac{\Gamma_{R}}{2} n_{R}^{(-)}(E 2)\left(\rho_{12}^{\left(n_{2}\right)}+\rho_{21}^{\left(n_{2}\right)}\right), \\
& \times(E 2)] \rho_{12}^{\left(n_{2}\right)} . \\
\dot{\rho}_{12}^{\left(n_{2}\right)}= & i \delta \epsilon \rho_{12}^{\left(n_{2}\right)}+\frac{1}{2} \Gamma_{L}\left[n_{L}^{(+)}(E 2)+n_{L}^{(+)}(E 1)\right] \rho_{00}^{\left(n_{2}\right)}+\frac{\eta}{2} \Gamma_{R}\left[n_{R}^{(+)}(E 1)+\Gamma_{R} n_{R}^{(-)}(E 1)+\Gamma_{L} n_{L}^{(-)}(E 2)+\Gamma_{R} n_{R}^{(-)}\right. \\
\dot{\rho}_{22}^{\left(n_{2}\right)}= & \Gamma_{L} n_{L}^{(+)}(E 2) \rho_{00}^{\left(n_{2}\right)}+\Gamma_{R} n_{R}^{(+)}(E 2) \rho_{00}^{\left(n_{2}+1\right)}-\left[\Gamma_{L} n_{L}^{(-)}(E 2)\right. \\
& \left.+\Gamma_{R} n_{R}^{(-)}(E 2)\right] \rho_{22}^{\left(n_{2}\right)}-\frac{\Gamma_{L}}{2} n_{L}^{(-)}(E 1)\left(\rho_{12}^{\left(n_{2}\right)}+\rho_{21}^{\left(n_{2}\right)}\right) \\
& -\eta \frac{\Gamma_{R}}{2} n_{R}^{(-)}(E 1)\left(\rho_{12}^{\left(n_{2}\right)}+\rho_{21}^{\left(n_{2}\right)}\right), \\
& (E 2) \rho_{22}^{\left(n_{2}\right)}-\frac{1}{2} \Gamma_{L}\left[n_{L}^{(-)}(E 1) \rho_{11}^{\left(n_{2}\right)}+n_{L}^{(-)}(E 2) \rho_{22}^{\left(n_{2}\right)}\right] \\
& \\
& \\
& \\
&
\end{aligned}
$$

Similarly to the case of zero temperature, we have summed $n_{1}$ and remained only $n_{2}$, indicating the FCS of electrons flowing into the drain reservoir. It would be of interest to note that the structure of the above equations differs from the one at zero temperature, i.e., Eq. (9). At finite temperature and small bias voltage, the electrons in the drain reservoir can jump back into the central dots. This backward process is described by the $\rho^{\left(n_{2}+1\right)}$ terms in the above equations, while these terms are absent in Eq. (9).

Based on Eq. (15) and the FCS formalism described in Sec. III, numerical results for finite temperature are plotted in

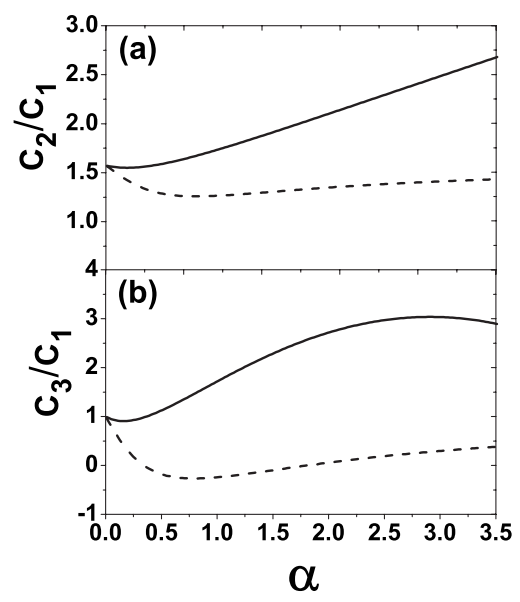

FIG. 4. Fano factor and normalized skewness versus $\alpha$ $\equiv \Gamma_{R} / \Gamma_{L}$ at finite temperatures. The solid and dashed curves display results for the constructive $(\eta=1)$ and destructive $(\eta=-1)$ interferences, respectively. Parameters: $E 2=-E 1=0.5, \Gamma_{L}=1.0, \mu_{L}=-\mu_{R}$ $=5.0$, and $\beta \equiv 1 / k_{B} T=0.15$.

Fig. 4. Compared to the zero temperature results, a few features are discussed as follows: (i) For the constructive interference $(\eta=1)$ at small values of $\alpha$, the thermal noise will enhance the Fano factor. This is because for small $\alpha$, the effective fast-and-slow channels cannot be developed, thus the thermal noise dominates the current fluctuations and leads to a super-Poisson behavior. This reasoning is also applicable to the entirely enhanced Fano factor of the destructive interference $(\eta=-1)$, which is shown by the dashed curve in Fig. 4(a). (ii) However, in the case of constructive interference $(\eta=1)$ and for those $\alpha$ where the effective fastand-slow channels are well developed, it is found that the thermal noise will reduce the Fano factor. In this regime, the thermal occupations around the Fermi levels of the electron reservoirs will degrade the effectiveness of the fast-and-slow channel based super-Poisson mechanism. As a result, the Fano factor is reduced. (iii) Owing to the involvement of thermal noise, the behavior of skewness changes considerably. The large negative skewness, which is accompanied with the constructive super-Poisson noise at zero temperature, will change its sign to positive by increasing temperature. In contrast, no considerable changes with temperature are observed for the skewness associated with the destructive interference.

\section{CONCLUSION}

To summarize, we have presented a FCS study for transport through a mesoscopic Coulomb blockade system with two symmetric channels. The FCS analysis showed that the shot noise and skewness are more sensitive than the average current to the quantum interference. For instance, in the symmetric case, the quantum constructive interference and the classical noninterference will lead to the same transport current, but different shot noise and skewness. Moreover, in the regime of strong Coulomb blockade, the interesting superPoisson behavior was found as a consequence of quantum 
constructive interference. By state-representation transformation, this phenomenon was understood as a result of the formation of effective fast-and-slow transport channels. Other results, such as the dephasing and the finite temperature effects, were also presented together with physical interpretations. Experiments within current technology can examine the predictions of this work.

Finally, the FCS approach employed in this work, based on the generalized particle-number-resolved master equation [cf. Eq. (5)], can account for not only the many-body Coulomb interaction ${ }^{29}$ but also the internal quantum coherence. In sequential tunneling regime, the approach is applicable to finite temperatures and small bias voltages. Further generalization to higher order tunneling processes and strongly nonMarkovian regime is of great interest and a challenging subject for future work.

\section{ACKNOWLEDGMENTS}

This work was supported by the National Natural Science Foundation of China under Grants No. 60425412 and No. 90503013, and the Research Grants Council of the Hong Kong Government.

\section{APPENDIX A}

In this appendix we present the explicit expressions of the spectral functions in Eq. (5) in the main text. For noninteracting electrodes and under the wide-band approximation, the reservoir correlation functions simply read

$$
\begin{array}{r}
C_{L i j}^{( \pm)}(t-\tau)=g_{L} \Omega_{L}^{2} \int d \varepsilon_{L k} e^{ \pm i \varepsilon_{L k}(t-\tau)} n_{L}^{( \pm)}\left(\varepsilon_{L k}\right), \quad(\mathrm{A} 1 \mathrm{a}) \\
C_{R i i}^{( \pm)}(t-\tau)=g_{R} \Omega_{R}^{2} \int d \varepsilon_{R k} e^{ \pm i \varepsilon_{R k}(t-\tau)} n_{R}^{( \pm)}\left(\varepsilon_{R k}\right), \quad(\mathrm{A} 1 \mathrm{~b}) \\
C_{R 12}^{( \pm)}(t-\tau)=\eta g_{R} \Omega_{R}^{2} \int d \varepsilon_{R k} e^{ \pm i \varepsilon_{R k}(t-\tau)} n_{R}^{( \pm)}\left(\varepsilon_{R k}\right)=C_{R 21}^{( \pm)}(t-\tau) .
\end{array}
$$

Here, the indices $i$ and $j$ denote the dot states $|1\rangle$ and $|2\rangle$. Since the two dot states are almost degenerate in energy and are equally coupled to the electrodes, in the above we have assumed that $\Omega_{i \alpha} \Omega_{j \alpha}^{*}=\Omega_{i \alpha}^{*} \Omega_{j \alpha}=\Omega_{\alpha}^{2}$. Note that an exception is $\Omega_{1 R}^{*} \Omega_{2 R}=\Omega_{2 R}^{*} \Omega_{1 R}=\eta \Omega_{R}^{2}$, where $\eta= \pm 1$ is the relative phase factor. This is because we have attributed the phase difference to the coupling amplitudes with the right electrodes. The electron and hole occupation functions are introduced, respectively, as $n_{\alpha}^{(+)}\left(\varepsilon_{\alpha k}\right)=n_{\alpha}\left(\varepsilon_{\alpha k}\right)$ and $n_{\alpha}^{(-)}\left(\varepsilon_{\alpha k}\right)=1-n_{\alpha}\left(\varepsilon_{\alpha k}\right)$, with $n_{\alpha}\left(\varepsilon_{\alpha k}\right)$ the Fermi function. Fourier transformation of Eqs. (A1) gives the spectral functions

$$
\begin{aligned}
& \widetilde{C}_{L i j}^{( \pm)}(\omega)=\Gamma_{L} n_{L}^{( \pm)}(\mp \omega), \\
& \widetilde{C}_{R i i}^{( \pm)}(\omega)=\Gamma_{R} n_{R}^{( \pm)}(\mp \omega),
\end{aligned}
$$

$$
\widetilde{C}_{R 12}^{( \pm)}(\omega)=\widetilde{C}_{R 21}^{( \pm)}(\omega)=\eta \Gamma_{R} n_{R}^{( \pm)}(\mp \omega),
$$

where $\Gamma_{\alpha}=2 \pi g_{\alpha} \Omega_{\alpha}^{2}$.

Furthermore, using $\mathcal{L} d_{j}=-\left(E_{j}+U n_{j}^{-}\right) d_{j}$, the operators $A_{\alpha j}^{( \pm)}$ in Eq. (5), which is defined by $A_{\alpha j}^{(+)}=\Sigma_{i} \widetilde{C}_{\alpha i j}^{(+)}(+\mathcal{L}) d_{i}, A_{\alpha j}^{(-)}$ $=\sum_{i} \widetilde{C}_{\alpha j i}^{(-)}(-\mathcal{L}) d_{i}$, are accordingly obtained as

$$
\begin{aligned}
& A_{\alpha j}^{(+)}=\sum_{i} \widetilde{C}_{\alpha i j}^{(+)}\left[-\left(E_{i}+U n_{i}^{-}\right)\right] d_{i}, \\
& A_{\alpha j}^{(-)}=\sum_{i} \widetilde{C}_{\alpha j i}^{(-)}\left[+\left(E_{i}+U n_{i}^{-}\right)\right] d_{i} .
\end{aligned}
$$

Here the index $\bar{i}$ simply means differing from $i$, i.e., $\bar{i}=2$ if $i=1$, and vice versa.

\section{APPENDIX B}

Via the transformation of state representation as described in the main text, the energy levels of the transformed dot states and their effective coupling strength read

$$
\begin{gathered}
\tilde{E}_{1}=\frac{\delta \epsilon}{2} \frac{\Omega_{R}^{2}-\Omega_{L}^{2}}{\Omega_{L}^{2}+\Omega_{R}^{2}}, \\
\tilde{E}_{2}=\frac{\delta \epsilon}{2} \frac{\Omega_{L}^{2}-\Omega_{R}^{2}}{\Omega_{L}^{2}+\Omega_{R}^{2}}, \\
\gamma=\delta \epsilon \frac{\Omega_{L} \Omega_{R}}{\Omega_{L}^{2}+\Omega_{R}^{2}} .
\end{gathered}
$$

Simple algebra also gives rise to the effective coupling strengths of the dot states with the electrodes

$$
\begin{aligned}
& \tilde{\Omega}_{1 L}=\frac{\Omega_{1 L}}{\sqrt{\Omega_{L}^{2}+\Omega_{R}^{2}}}\left(\Omega_{L}+\Omega_{R}\right), \\
& \tilde{\Omega}_{1 R}=\frac{\Omega_{2 R}}{\sqrt{\Omega_{L}^{2}+\Omega_{R}^{2}}}\left(\eta \Omega_{L}+\Omega_{R}\right), \\
& \tilde{\Omega}_{2 L}=\frac{\Omega_{1 L}}{\sqrt{\Omega_{L}^{2}+\Omega_{R}^{2}}}\left(\Omega_{L}-\Omega_{R}\right), \\
& \widetilde{\Omega}_{2 R}=\frac{\Omega_{2 R}}{\sqrt{\Omega_{L}^{2}+\Omega_{R}^{2}}}\left(\Omega_{L}-\eta \Omega_{R}\right) .
\end{aligned}
$$

More transparently, for $\eta=1$, the corresponding tunneling rates read

$$
\begin{gathered}
\Gamma_{1 L}=\frac{\Gamma_{L}\left(\Gamma_{L}+\Gamma_{R}+2 \sqrt{\Gamma_{L} \Gamma_{R}}\right)}{\Gamma_{L}+\Gamma_{R}}, \\
\Gamma_{1 R}=\frac{\Gamma_{R}\left(\Gamma_{L}+\Gamma_{R}+2 \sqrt{\Gamma_{L} \Gamma_{R}}\right)}{\Gamma_{L}+\Gamma_{R}}, \\
\Gamma_{2 L}=\frac{\Gamma_{L}\left(\Gamma_{L}+\Gamma_{R}-2 \sqrt{\Gamma_{L} \Gamma_{R}}\right)}{\Gamma_{L}+\Gamma_{R}},
\end{gathered}
$$




$$
\Gamma_{2 R}=\frac{\Gamma_{R}\left(\Gamma_{L}+\Gamma_{R}-2 \sqrt{\Gamma_{L} \Gamma_{R}}\right)}{\Gamma_{L}+\Gamma_{R}},
$$

and for $\eta=-1$, they are

$$
\Gamma_{1 L}=\frac{\Gamma_{L}\left(\Gamma_{L}+\Gamma_{R}+2 \sqrt{\Gamma_{L} \Gamma_{R}}\right)}{\Gamma_{L}+\Gamma_{R}},
$$

*xqli@red.semi.ac.cn

${ }^{1}$ Ya. M. Blanter and M. Büttiker, Phys. Rep. 336, 1 (2000); Quantum Noise in Mesoscopic Physics, edited by Yu. V. Nazarov (Kluwer, Dordrecht, 2003).

${ }^{2}$ L. S. Levitov, H. W. Lee, and G. B. Lesovik, J. Math. Phys. 37, 4845 (1996).

${ }^{3}$ V. K. Khlus, Sov. Phys. JETP 66, 1243 (1987).

${ }^{4}$ S. Gustavsson, R. Leturcq, B. Simovič, R. Schleser, T. Ihn, P. Studerus, K. Ensslin, D. C. Driscoll, and A. C. Gossard, Phys. Rev. Lett. 96, 076605 (2006); T. Fujisawa, T. Hayashi, R. Tomita, and Y. Hirayama, Science 312, 1634 (2006).

${ }^{5}$ M. Büttiker, Phys. Rev. B 46, 12485 (1992); M. Henny, S. Oberholzer, C. Strunk, and C. Schönenberger, Science 284, 296 (1999); W. D. Oliver, Jungsang Kim, Robert C. Liu, and Y. Yamamoto, ibid. 284, 299 (1999).

${ }^{6}$ M. J. M. de Jong and C. W. J. Beenakker, Phys. Rev. B 49, 16070 (1994); B. A. Muzykantskii and D. E. Khmelnitskii, ibid. 50, 3982 (1994); W. Belzig and Yu. V. Nazarov, Phys. Rev. Lett. 87, 067006 (2001).

${ }^{7}$ A. A. Kozhevnikov, R. J. Schoelkopf, and D. E. Prober, Phys. Rev. Lett. 84, 3398 (2000); X. Jehl, M. Sanquer, R. Calemczuk, and D. Mailly, Nature (London) 405, 50 (2000).

${ }^{8}$ T. Hoss, C. Strunk, T. Nussbaumer, R. Huber, U. Staufer, and C. Schönenberger, Phys. Rev. B 62, 4079 (2000); R. Cron, M. F. Goffman, D. Esteve, and C. Urbina, Phys. Rev. Lett. 86, 4104 (2001).

${ }^{9}$ J. C. Cuevas, A. Martín-Rodero, and A. L. Yeyati, Phys. Rev. Lett. 82, 4086 (1999); Y. Naveh and D. V. Averin, ibid. 82, 4090 (1999).

${ }^{10}$ W. Belzig and Yu. V. Nazarov, Phys. Rev. Lett. 87, 197006 (2001)

${ }^{11}$ G. Johansson, P. Samuelsson, and Åke Ingerman, Phys. Rev. Lett. 91, 187002 (2003); S. Pilgram and P. Samuelsson, ibid. 94, 086806 (2005).

${ }^{12}$ S. S. Safonov, A. K. Savchenko, D. A. Bagrets, O. N. Jouravlev, Yu. V. Nazarov, E. H. Linfield, and D. A. Ritchie, Phys. Rev. Lett. 91, 136801 (2003).

${ }^{13}$ A. Thielmann, M. H. Hettler, J. König, and G. Schön, Phys. Rev. B 71, 045341 (2005); J. Aghassi, A. Thielmann, M. H. Hettler, and G. Schön, ibid. 73, 195323 (2006).

${ }^{14}$ W. Belzig, Phys. Rev. B 71, 161301(R) (2005).

${ }^{15}$ B. R. Bulka, Phys. Rev. B 62, 1186 (2000); A. Cottet, W. Belzig, and C. Bruder, Phys. Rev. Lett. 92, 206801 (2004).

${ }^{16}$ G. Iannaccone, G. Lombardi, M. Macucci, and B. Pellegrini, Phys. Rev. Lett. 80, 1054 (1998); A. N. Jordan and E. V. Sukho- rukov, ibid. 93, 260604 (2004); 94, 059901(E) (2005).

${ }^{17}$ E. V. Sukhorukov, G. Burkard, and D. Loss, Phys. Rev. B 63, 125315 (2001); A. Thielmann, M. H. Hettler, J. König, and G. Schön, Phys. Rev. Lett. 95, 146806 (2005).

${ }^{18}$ E. Onac, F. Balestro, B. Trauzettel, C. F. J. Lodewijk, and L. P. Kouwenhoven, Phys. Rev. Lett. 96, 026803 (2006).

${ }^{19}$ Ya. M. Blanter, O. Usmani, and Yu. V. Nazarov, Phys. Rev. Lett. 93, 136802 (2004); 94, 049904(E) (2005); T. Novotny, A. Donarini, C. Flindt, and A.-P. Jauho, ibid. 92, 248302 (2004); J. Koch and Felix von Oppen, ibid. 94, 206804 (2005).

${ }^{20}$ C. W. Groth, B. Michaelis, and C. W. J. Beenakker, Phys. Rev. B 74, 125315 (2006).

${ }^{21}$ S. A. Gurvitz, IEEE Trans. Nanotechnol. 4, 45 (2005).

${ }^{22}$ A. W. Holleitner, C. R. Decker, H. Qin, K. Eberl, and R. H. Blick, Phys. Rev. Lett. 87, 256802 (2001); A. W. Holleitner, R. H. Blick, A. K. Hüttel, K. Eberl, and J. P. Kotthaus, Science 297, 70 (2002).

${ }^{23}$ M. Sigrist, T. Ihn, K. Ensslin, D. Loss, M. Reinwald, and W. Wegscheider, Phys. Rev. Lett. 96, 036804 (2006).

${ }^{24}$ G. Hackenbroich, Phys. Rep. 343, 463 (2001) and references there in.

${ }^{25}$ A. Yacoby, M. Heiblum, D. Mahalu, and H. Shtrikman, Phys. Rev. Lett. 74, 4047 (1995); R. Schuster, E. Buks, M. Heiblum, D. Mahalu, V. Umansky, and H. Shtrikman, Nature (London) 385, 417 (1997); M. Avinun-Kalish, M. Heiblum, O. Zarchin, D. Mahalu, and V. Umansky, ibid. 436, 529 (2005).

${ }^{26}$ O. Entin-Wohlman, A. Aharony, Y. Imry, Y. Levinson, and A. Schiller, Phys. Rev. Lett. 88, 166801 (2002); A. Aharony, O. Entin-Wohlman, B. I. Halperin, and Y. Imry, Phys. Rev. B 66, 115311 (2002).

${ }^{27}$ X.-Q. Li, P. Cui, and Y. J. Yan, Phys. Rev. Lett. 94, 066803 (2005).

${ }^{28}$ X.-Q. Li, J. Luo, Y.-G. Yang, P. Cui, and Y. J. Yan, Phys. Rev. B 71, 205304 (2005).

${ }^{29}$ D. A. Bagrets and Yu. V. Nazarov, Phys. Rev. B 67, 085316 (2003).

${ }^{30}$ S. A. Gurvitz and Ya. S. Prager, Phys. Rev. B 53, 15932 (1996).

${ }^{31}$ Yu. V. Nazarov, Ann. Phys. (Leipzig) 8, SI-193 (1999); W. Belzig, in Quantum Noise in Mesoscopic Physics, edited by Yuli. V. Nazarov (Kluwer, Dordrecht, 2003), p. 463.

${ }^{32}$ Yu. V. Nazarov, in Quantum Dynamics of Submicron Structures, edited by H. Cerdeira, B. Kramer, and G. Schoen (Kluwer, Dordrecht, 1995), p. 687; Yu. V. Nazarov and D. A. Bagrets, Phys. Rev. Lett. 88, 196801 (2002).

${ }^{33}$ C. Flindt, T. Novotny, and A. P. Jauho, Europhys. Lett. 69, 475 
(2005).

${ }^{34}$ G. Kießlich, P. Samuelsson, A. Wacker, and E. Schöll, Phys. Rev. B 73, 033312 (2006).

${ }^{35}$ Rui-Xue Xu, Ping Cui, Xin-Qi Li, Yan Mo, and YiJing Yan, J. Chem. Phys. 122, 041103 (2005).

${ }^{36}$ Jinshuang Jin, Sven Welack, JunYan Luo, Xin-Qi Li, Ping Cui, Rui-Xue Xu, and YiJing Yan, J. Chem. Phys. 126, 134113
(2007); Rui-Xue Xu and YiJing Yan, Phys. Rev. E 75, 031107 (2007).

${ }^{37}$ Ping Cui, Xin-Qi Li, Jiushu Shao, and YiJing Yan, Phys. Lett. A 357, 449 (2006).

${ }^{38}$ S. A. Gurvitz, Phys. Rev. B 56, 15215 (1997).

${ }^{39}$ B. Michaelis, C. Emary, and C. W. J. Beenakker, Europhys. Lett. 73, 677 (2006). 